\title{
Development and Updating of 3D GIS by Using Aerial Photography Systems
}

\author{
Kirill Zhigalov ${ }^{1,2,}{ }^{*}$, Denis Efremov ${ }^{2}$, Gregory Bubnov ${ }^{2,3}$ and Evgeniy Pluzhnik ${ }^{2}$ \\ ${ }^{1}$ V.A. Trapeznikov institute of Control Science of Russian Academy of Sciences, 117997 Moscow, Russia \\ ${ }^{2}$ Moscow Technological Institute, 119334 Moscow, Russia \\ ${ }^{3}$ Moscow Institute of Physics and Technology, 141701 Dolgoprudny, Moscow region, Russia
}

\begin{abstract}
In the article the main opportunities for engineers at the GIS creation/updating of systems by means of multicopter aerial photography systems are described. Usage of the methods described in the article will allow increasing the speed and informational content of the GIS construction/updating at rather small labor costs. Besides, the described automation of the processes of filming from the unmanned aerial vehicle will allow to supplement three-dimensional models of objects in GIS with earlier inaccessible data.
\end{abstract}

\section{Introduction}

Now geographic information systems are widespread in all fields of personal activity. The main requirements for them are efficiency of updating and accuracy of the data containing in them. The basis of all geographic information systems are different cartographic materials (aerial photography, maps, plans and other) describing the area. The area constantly changes as due to activities of the person (development of pits, deforestation, any construction), and due to natural processes (growth of trees, degradation of the rivers and other) [1-3].

In connection with the aforesaid, expeditious updating of GIS is rather an important task.

It should be noted that 3D GIS also includes 3D models of the area (columns, structures, bridges and other). These models, in case of need are also a subject for updating (see Figure 1).

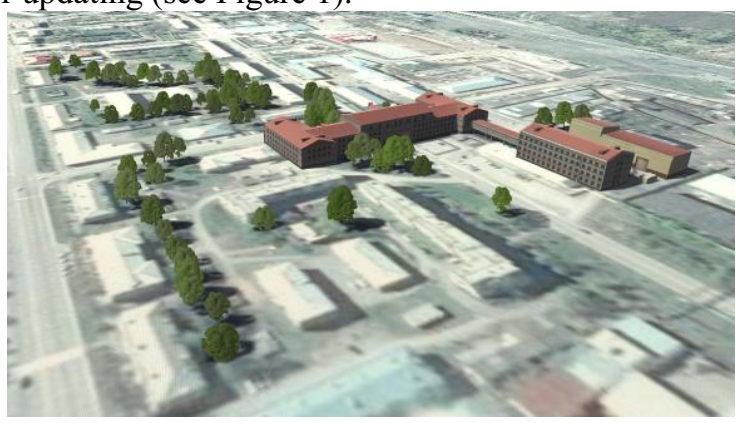

Fig. 1. An example of the 3D screen of GIS

\section{Standard ways of the GIS construction/updating}

Updating of these 3D GIS-systems requires obtaining coordinates of all changes on the area in any kind and pictures of the objects of the area taken from the different parties at right angle to the copter [4].

Nowadays for the purpose of data acquisition for the GIS updating 2 main methods are used:

- data acquisition in the manual way (a round of all the territory manually with special devices);

- data acquisition by means of earth remote-sensing instruments, generally they are laser scanners and cameras installed on different carriers (satellites, planes, cars).

The first way is basically used when updating small territory of GIS (up to several hectares).

The second method is applicable in case of big areas of a covering of GIS (coal pits, the woods, roads, lines of the power line, cities and so on).

However both of the ways are widely used, they have a number of disadvantages.

While using a manual way of updating GIS, people can only fix the position of objects on the area without a possibility of addition in GIS pictures of a surface, which allows manufacturing only plans/maps.

In case of manufacturing models of difficult objects (see Figure 2) a person can't see some parts (i.e. upper ones), even using difficult devices, such as, the $3 \mathrm{D}$ laser scanner.

The last makes an influence for quality of models and their accuracy.

In case of data collection by remote-sensing instruments we can make rather accurate images of the area (aerial photography, see Figure 3) and coordinates of points of a surface (when using 3D laser scanners).

Nevertheless, for production of 3D models of the objects it is necessary to use the pictures made from different foreshortenings and not just from above (see Figure 4.)

\footnotetext{
Corresponding author: kshakalov@mail.ru
} 


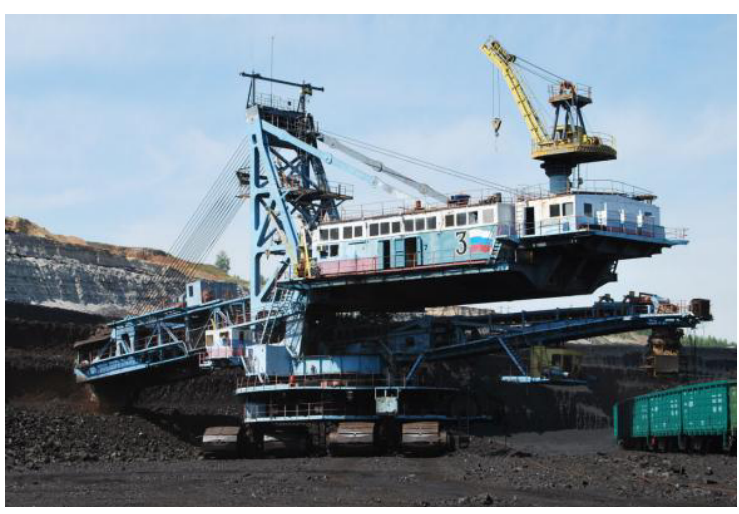

Fig. 2. An example of a difficult object

Besides, receipt of such data is rather costly from the financial point of view.

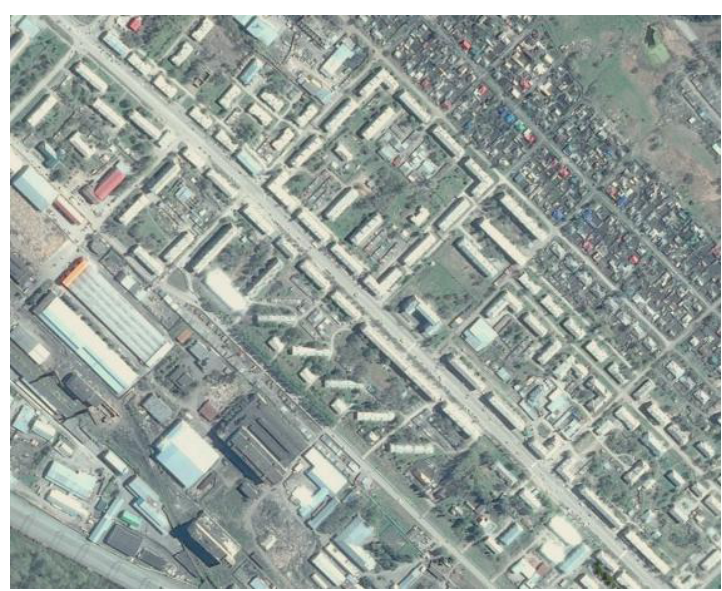

Fig. 3. An example of the result of aerial photography

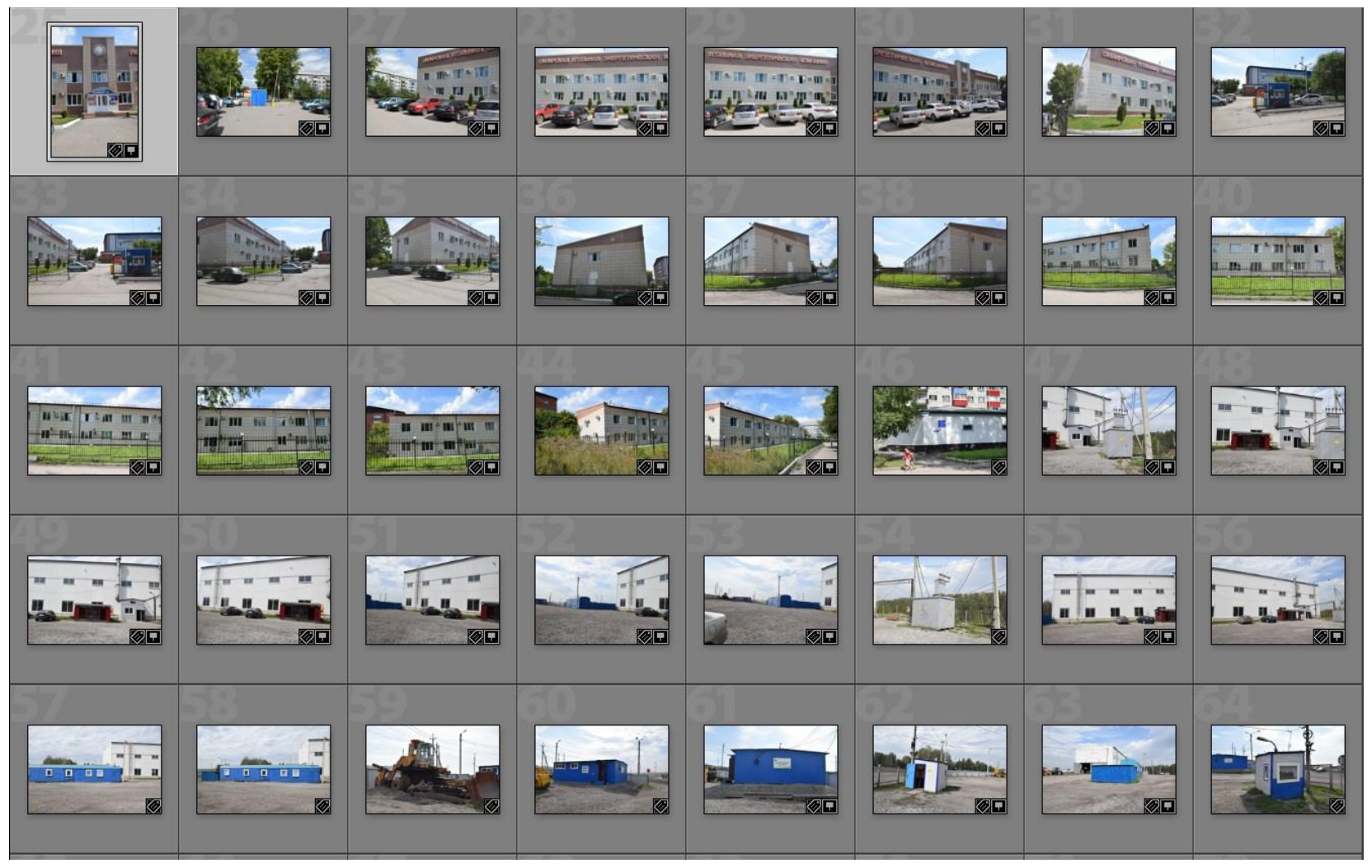

Fig. 4. An example of an image of one object from different foreshortenings

Such suspension allows changing position of the

\section{Possible improvements connected with the application of copter in aerial photography}

It is possible to integrate the methods described above in one universal method with the use of modern quad- and hexacopters, with dynamically suspended cameras (see Figure 5). One can use remotely operated small helicopters for this purpose, but the vibration on one motor is rather big for this purpose, and that may affect the quality of final images. In this connection it is recommended to use quad- and hexacopter based systems [5]. camera remotely on a copter system from parallel to the horizon (for shootings of objects of a terrain, see Figure 6) to perpendicular (for shooting of a surface, see Figure 7).

Modern copters allow moving away from the operator on the distance up to 7 kilometres and on the height up to 5000 meters, which allows covering the area of photography up to 140 square kilometres with one copter.

This configuration allows taking pictures of separate objects located at the distance up to 6 kilometers from the operator from all necessary foreshortenings that leads to obtaining necessary information about remote objects quickly. 


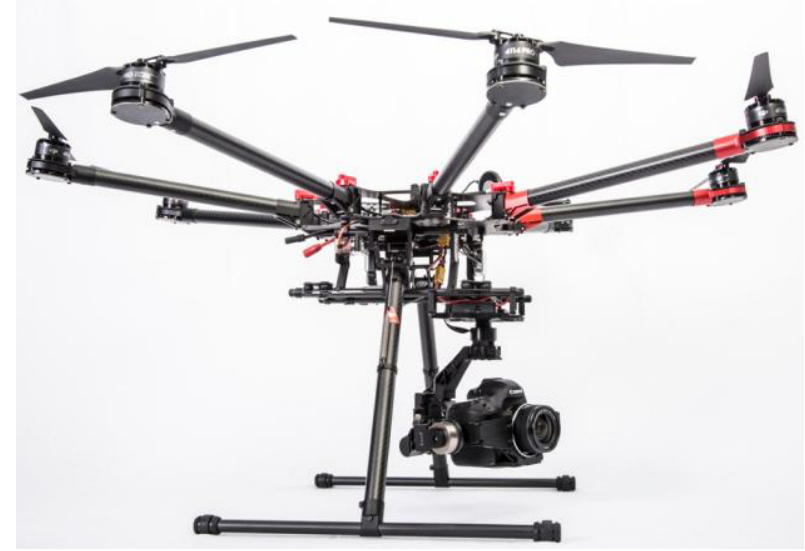

Fig. 5. An example of a copter with a dynamic suspension of a camera

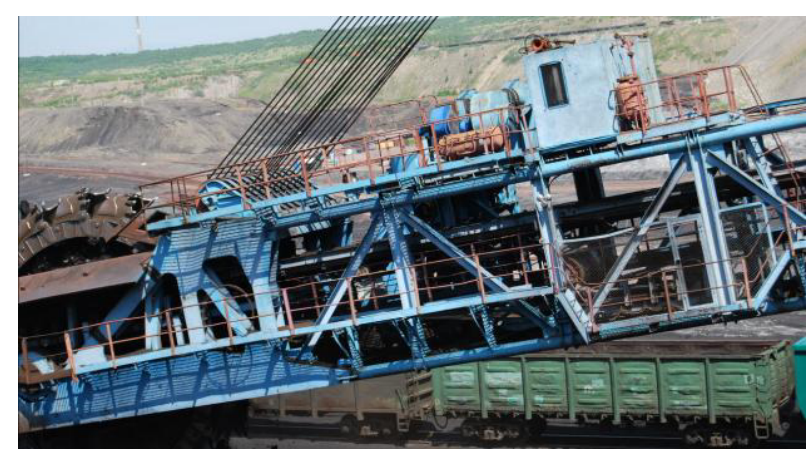

Fig. 6. An example of the image of an object for modeling captured by a copter

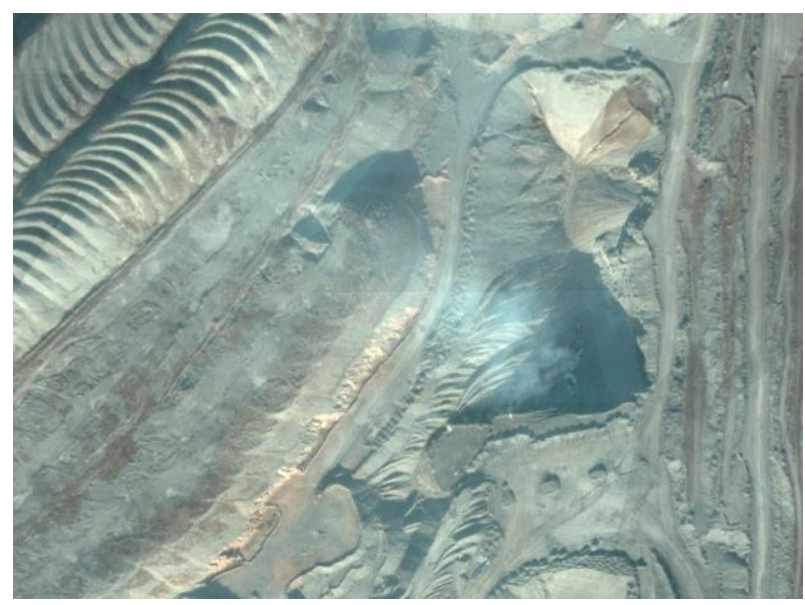

Fig. 7. An example of the image of a surface captured a copter

Nevertheless, there are several disadvantages of data acquisition by means of copter:

- rather big weight of a dynamic suspension with the stabilizer and the camera reduces maneuverability and time of the device usage;

- rather small height of work (in comparison with satellites and the air planes) that leads to a rather large number of pictures of a surface, that causes difficulties at their storage and processing;

- helplessness of modern civil copters in front of the systems of muffling of a signal of management.
The first two difficulties are solved by using modern accumulators with powerful motors, nevertheless they do not strongly influence the final result.

The last difficulty is solved on some copters with the help of automatic return systems and sensors of provision of surrounding objects.

Nevertheless, it is possible to use systems of automated management with the help of integration of digital on-board computer maps and laying of the automated routes of a returning.

As a test attempt of such decision GIS suek3d with a prepared digital district map in advance has been used. The copter several times passed along in advance set flight line (see Figure 8).

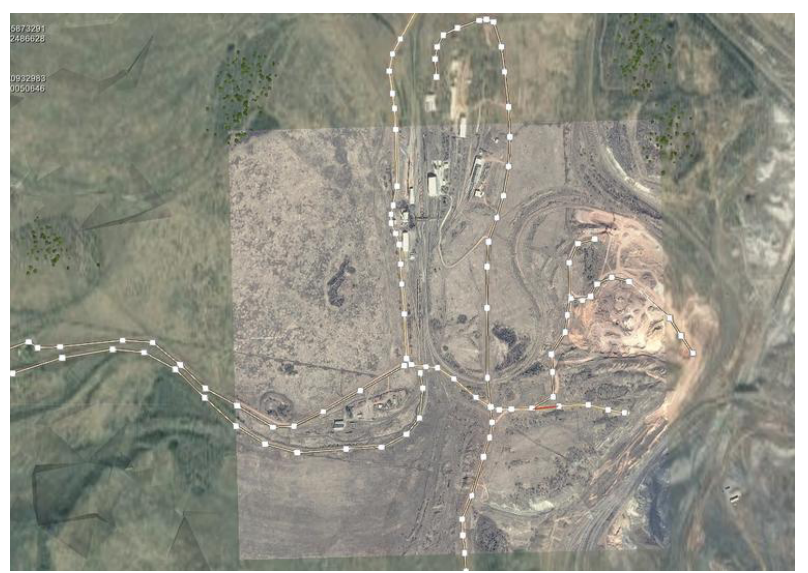

Fig. 8. An example of a modelling flight line in GIS

The test realization on the copter consisted of a control system on the basis of a phone with the MS Windows 10 operating system and a simple code, written on $\mathrm{C}++$. The data have been transferred to the control system by means of the table with $\mathrm{X}, \mathrm{Y}, \mathrm{Z}$ coordinates for each point of a flight line.

It should be noted, that the system reduced the payload by 200 grams and the flight time by 5 minutes. In such realization it is possible to use less qualified personnel for starts.

\section{Conclusion}

Updating of 3D GIS data is an important and universal task. Three-dimensional GIS data differ from usual ones by a large number of models of objects and the equipment. Modern ways of data acquisition for the GIS updating are well adapted for updating of standard GISsystems, nevertheless they have a number of the disadvantages complicating their use in threedimensional GIS. Copters with small additions in a design are suitable for the new generation GIS updating. The offered option of the usage of a dynamic suspension significantly expands the possibilities of a usual copter and allows to use it as an absolutely new universal platform for data collection for the GIS updating and to accelerate this process. It is especially important in the countries where data collection is limited by climatic conditions. 


\section{References}

1. A. Bhardwaja, L. Samb, Akankshad, F.J. MartínTorresa, R. Kumarb, Remote Sensing of Environment, 175, 196-204 (2016)

2. K. Zhigalov, Usage of the game technologies engines for the purpose of modern geographic information systems creation, ITM Web of Conferences, 6, 03018 (2016)
3. E. Pluzhnik; E. Nikulchev, Virtual Laboratories in Cloud Infrastructure of Educational Institutions 2014 2nd International Conference on Emission Electronics (ICEE), 67-69 (2014)

4. K. Zhigalov, Cloud of Science, 3, 71-80 (2016)

5. In-Ae Yeoa, Jurng-Jae Yee. Automation in Construction, 71, 398-413 (2016) 